\title{
Equivalent Realization of Cylindrical Electromagnetic Concentrator Based On Transmission Line Network
}

\author{
T.H. Li \\ Wireless Innovation Lab \\ School of Information Science and Engineering \\ Yunnan University \\ Kunming 650091, China \\ M. Huang* \\ Wireless Innovation Lab \\ School of Information Science and Engineering \\ Yunnan University \\ Kunming 650091, China
}

\author{
Y. Su \\ School of Information and Electronics \\ Beijing Institute of technology \\ Beijing 100081, China \\ J.J. Yang \\ Wireless Innovation Lab \\ School of Information Science and Engineering \\ Yunnan University \\ Kunming 650091, China
}

\author{
X.G. Xu \\ Wireless Innovation Lab \\ School of Information Science and Engineering \\ Yunnan University \\ Kunming 650091, China
}

\begin{abstract}
Electromagnetic concentrator as an important wavemanipulation device plays important roles in receiving antenna, solar cell or similar devices. However, anisotropic and inhomogeneous material properties seriously impede the practical fabrication of the device. In this paper, an equivalent realization method for the cylindrical concentrator is proposed based on the transmission line (TL) network. The effectiveness of the method is validated through the Agilent's Advanced Design System (ADS) circuit simulator, and the performance of presented TL concentrator is compared with the ideal concentrator. Besides, the broadband characteristics and the robustness of TL concentrator against the material loss of circuit elements are also discussed. This work expands the application of TL theory and provides a feasible solution to push the concentrator much closer to the application.
\end{abstract}

Keywords-electromagnetic concentrator; TL network; transformation optics

\section{INTRODUCTION}

As an emerging research field, transformation optics (TO) has recently created a great sensation in the scientific community since it provide us with unprecedented freedom to design novel wave-manipulation devices that seemed unconceivable a decade ago [1-5], such as invisible cloaks, perfect lenses, and electromagnetic concentrators. Among these astonishing devices, concentrators capable of focusing electromagnetic wave into a core region to get high energy density have aroused lots of interest due to its broad application prospects in designing efficient receiving antenna, solar cell or similar devices [6-12]. The first electromagnetic concentrator with cylindrical shape is proposed by Rahm et al [6], and then intensive studies on the concentrator have been conducted, including coneshaped concentrators [7], arbitrary-shaped concentrators [8], non-rotationally invariant concentrators [9], homogeneousmaterials-constructed concentrators [10], concentrators with only axial parameter spatially variant [11], and concentrators with minimized scattering [12]. The foregoing investigations are really attractive, but the material parameters of aforesaid concentrators are usually anisotropic and/or inhomogeneous. It's worth noting that the concentrators with such parameter distributions can not be directly implemented with the naturally available materials, and thus to seek an equivalent realization method is of great significance.

In this paper, a TL circuit method to achieve the cylindrical concentrator with anisotropic and inhomogeneous material parameters is presented. Diagonal components of TO-based parameters in each TL unit cell are equivalently realized by four inductors in series and one grounded capacitor in shunt. Due to the nonresonant nature of TL network, the weak dispersion of materials is introduced which results in the broadband characteristic of the proposed TL concentrator. Simulations based on ADS software are performed to verify the feasibility of suggested method and the performance of designed concentrator. Furthermore, the effect of material loss of circuit elements on the performance of TL concentrator is also analyzed.

\section{MODEL AND METHOD}

Figure 1 shows the basic spatial transformation for cylindrical electromagnetic concentrator, in which Figs. 1(a) 
and 1(b) correspond to the virtual and real spaces. As suggested by Rahm et al [6], a two-step spatial transformation is necessary for designing such a device. In the first step, the region $r^{\prime} \in\left[R_{2}, R_{3}\right]$ is expanded into a shell region $r \in\left[R_{1}, R_{3}\right]$ along the radial direction, while the angular and axial directions experience no changes. The transformation in the second step is similar, but the region $r^{\prime} \in\left[0, R_{2}\right]$ is compressed into a core region $r \in\left[0, R_{1}\right]$. Assuming that the transformation equations between the virtual space $\left(r^{\prime}, \theta^{\prime}, z^{\prime}\right)$ and the real space $(r, \theta, z)$ are linear functions and following the spatial transformation outlined above, the transformation equations for each region can be easily obtained as

$$
\begin{gathered}
r^{\prime}=f(r)=\left\{\begin{array}{cc}
k r & 0 \leq r \leq R_{1} \\
k_{1} r+k_{2} & R_{1} \leq r \leq R_{3} \\
r & r \geq R_{3}
\end{array}\right. \\
\theta^{\prime}=\theta, z^{\prime}=z \\
\text { Where } k=R_{2} / R_{1} \quad, \quad k_{1}=\left(R_{3}-R_{2}\right) /\left(R_{3}-R_{1}\right) \quad \text { and }
\end{gathered}
$$
$k_{2}=\left(R_{2}-R_{1}\right) R_{3} /\left(R_{3}-R_{1}\right)$.

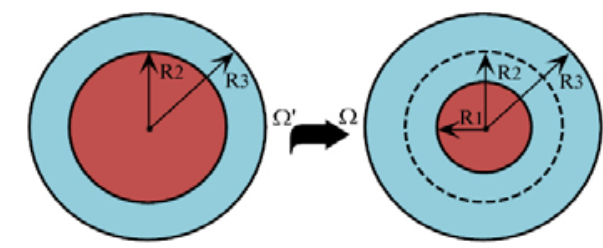

FIGURE I.BASIC SPATIAL TRANSFORMATION FOR THE CYLINDRICAL ELECTROMAGNETIC CONCENTRATOR, (A) VIRTUAL SPACE AND (B) REAL SPACE.

Without loss of generality, we take the transverse electric (TE) polarization mode as an example to start our discussion, in which electric field is polarized in the axial direction, and only $\mu_{r}, \mu_{\theta}, \varepsilon_{z}$ components of material parameters in cylindrical coordinates are relevant. Then according to TO theory, the effective material parameters are expressed as

$$
\begin{gathered}
\mu_{\text {corr }}=\mu_{b}, \mu_{\text {cor } \theta}=\mu_{b}, \varepsilon_{\text {corz }}=k^{2} \varepsilon_{b} \text { (2) } \\
\mu_{\text {midr }}=\frac{k_{1} r+k_{2}}{k_{1} r} \mu_{b}, \mu_{\text {mid } \theta}=\frac{k_{1} r}{k_{1} r+k_{2}} \mu_{b}, \varepsilon_{\text {midz }}=\frac{k_{1}\left(k_{1} r+k_{2}\right)}{r} \varepsilon_{b} \text { (3) } \\
\mu_{\text {bacr }}=\mu_{b}, \mu_{\text {bac } \theta}=\mu_{b}, \varepsilon_{\text {bacz }}=\varepsilon_{b}
\end{gathered}
$$

Where $\varepsilon_{b}$ and $\mu_{b}$ are the permittivity and permeability of the virtual space, the subscripts cor and mid denotes the core region $r \in\left[0, R_{1}\right]$ and the shell region $r \in\left[R_{1}, R_{3}\right]$ of cylindrical electromagnetic concentrator, and the subscript bac represent the background medium region $r \in\left[R_{3}, \infty\right]$. Eqs. (2)- (4) provide the general expressions of material parameters for the cylindrical concentrator and the background medium. It is not difficult to find that the required materials for the concentrator cannot be implemented with naturally available materials because of anisotropy and in homogeneity, which is induced by the spatial transformation along the radial direction. Therefore, it has important significance to explore an equivalent realization method for the concentrator in order to accelerate its practical application. As has been verified by the previous work [13, 14], the anisotropic inhomogeneous material parameters can be well approximated by a TL network based on the analogy of electromagnetic and TL theories as long as the size of each TL unit cell is much smaller than the wavelength. TL network can provide a relatively simple yet broadband solution. Next, we will focus on how to realize the cylindrical electromagnetic concentrator using TL network.

For the sake of simulating convenience, both the concentrator and the background medium are mimicked by the TL network so that the propagation property inside and outside the concentrator can be completely represented by the voltage distributions which is easy to be extracted. TL model is illustrated in Fig. 2, in which the whole network is divided into the small unit cells by a set of concentric circles and radial lines. The inset is a magnification of a single unit cell composed of four inductors in series and one grounded capacitor in shunt. By mapping the Maxwell equations into the Telegraph equations, the relations

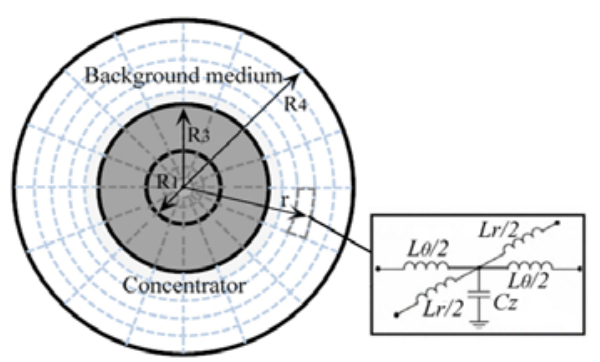

FIGURE II. TL MODEL FOR THE CONCENTRATOR AND THE BACKGROUND MEDIUM. THE INSET IS A MAGNIFICATION OF A SINGLE TL UNIT CELL COMPRISING FOUR INDUCTORS IN SERIES AND ONE GROUNDED CAPACITOR IN SHUNT.

Between the TO-based effective material parameters (i.e., $\mu_{r}, \mu_{\theta}$ and $\varepsilon_{z}$ ) and the TL parameters (i.e., $L_{r}, L_{\theta}$ and $C_{z}$ ) is derived as

$$
\begin{array}{r}
L_{r}(n)=\frac{\Delta r_{n}}{r_{n} \Delta \theta_{n}} \mu_{\theta}(n) d \\
L_{\theta}(n)=\frac{r_{n} \Delta \theta_{n}}{\Delta r_{n}} \mu_{r}(n) d \\
C_{z}(n)=\frac{r_{n} \Delta \theta_{n} \Delta r_{n}}{d} \varepsilon_{z}(n)
\end{array}
$$

Where the subscript $n$ represents the nth layer, $d$ is the thickness along the axial direction, and $r_{n}, \Delta r_{n}, \Delta \theta_{n}$ denotes the radial distance, the thickness and the span angle of unit cell in the nth layer, respectively. Substituting Eqs. (2)- (4) into Eq. (5), the corresponding TL parameters are found to be

$$
L_{\text {corr }}(n)=\mu_{b} \frac{\Delta r_{n}}{r_{n} \Delta \theta_{n}} d, L_{\text {core }}(n)=\mu_{b} \frac{r_{n} \Delta \theta_{n}}{\Delta r_{n}} d, C_{\text {corz }}(n)=\frac{\varepsilon_{b} k^{2} r_{n} \Delta \theta_{n} \Delta r_{n}}{d}
$$

for the core region of cylindrical electromagnetic 
concentrator,

$$
\begin{array}{r}
L_{\text {midr }}(n)=\mu_{b} \frac{k_{1} \Delta r_{n}}{\tau_{n} \Delta \theta_{n}} d \\
L_{\text {mid } \theta}(n)=\mu_{b} \frac{\tau_{n} \Delta \theta_{n}}{k_{1} \Delta r_{n}} d \\
C_{\text {midz }}(n)=\frac{\varepsilon_{b} \tau_{n} k_{1} \Delta \theta_{n} \Delta r_{n}}{d}
\end{array}
$$

For the shell region of the concentrator in which $\tau_{n}=k_{1} r_{n}+k_{2}$, and

$$
L_{\text {bacr }}(n)=\mu_{b} \frac{\Delta r_{n}}{r_{n} \Delta \theta_{n}} d, L_{\text {bac } \theta}(n)=\mu_{b} \frac{r_{n} \Delta \theta_{n}}{\Delta r_{n}} d, C_{\text {bacz }}(n)=\frac{\varepsilon_{b} r_{n} \Delta \theta_{n} \Delta r_{n}}{d} \text { (8) }
$$

For the background medium region. It should be noted that all the radial inductors for the inner boundary of concentrator are connected with each other to create an effect of a continuous boundary, whereas those for the outer boundary of background medium truncated by a circle with a radius of $R_{4}$ are terminated with the Bloch impedances [15] to achieve the match absorption and to mimic the infinite extended background. In our design, the geometric parameters of the concentrator are selected as $3 R_{1}=1.5 R_{2}=R_{3}=1.5 \lambda$, the truncated background region has a radius of $R_{4}=3 \lambda$, and the thickness along the axial direction is $d=\lambda / 10$. Here, $\lambda$ refers to incident wavelength at work frequency $f=50 \mathrm{MHz}$. The material parameters of virtual space are set as $\varepsilon_{b}=10^{-3} \varepsilon_{0}$ and $\mu_{b}=10^{7} \mu_{0}$, in which $\varepsilon_{0}$ and $\mu_{0}$ are the permittivity and permeability of free space. We benefit from such a choice is that the whole TL network is reduced to a reasonable dimension since the wavelength becomes $\lambda=6 \mathrm{~cm}$ under this circumstance. To well satisfy the longwavelength approximation, the concentric layers of TL network used to realize the core region, the shell region of the concentrator and the background medium region are all 15 layers. Each layer is equally divided into 90 unit cells along the angular direction, causing $4^{\circ}$ span angle for each unit cell. By combining the above conditions with Eqs. (2)(4) and Eqs. (6)- (8), the TO-based effective material parameters for the concentrator and the background medium region and the TL parameters applied to implement them are obtained, as portrayed by the blue solid and red dotted lines in Fig. 3. Obviously, the values of the indicators and capacitor are easily available in practice. In what follows, the effectiveness of TL parameters and the performance of designed concentrator are validated based on ADS circuit simulator.



FIGURE III. TO-BASED EFFECTIVE MATERIAL PARAMETERS (BLUE SOLID LINE) AND CORRESPONDING TL PARAMETERS (RED DOTTED LINE) FOR THE CONCENTRATOR AND THE BACKGROUND MEDIUM.

\section{SIMULATION RESULTS AND DISCUSSION}

In the simulation, the equivalent TL circuit for the concentrator and the background medium is built on the basis of ADS platform, and the quality factor for all circuit elements is set to $\mathrm{Q}=50$ to introduce a certain material loss. It should be pointed out that only a set of unit cells need to be firstly specified along the radial direction in the process of modeling owing to the rotational symmetry of TL network, and then we extend them to the whole region along the angular directions. Figures 4(b) depicts the voltage distributions of the equivalent TL circuit with the concentrator, in which a $50 \mathrm{MHz}$ point source is placed at the topside to imitate the cylindrical wave. As a comparison, the voltage distributions of TL circuit without the concentrator are also given out in Fig. 4(a). We can see from Fig. 4(b) that the cylindrical wave is perfectly focused into the core region when passing through the concentrator. So as to provide more convincing evidence, the electric field distributions in the vicinity of concentrator with the ideal TO-based material parameters are also simulated through the finite element software COMSOL, as shown in Fig. 4(c). The computational domain in Figs. 4(c) is surrounded by a cylindrical perfectly matched layer with a thickness of $2 \mathrm{~cm}$ which plays a similar role as the Bloch impedance. Comparing Fig. 4(b) with Fig. 4(c), it is evident that the voltage distributions are in good agreement with the electric field distributions. Hence the feasibility of implementing the cylindrical electromagnetic concentrator with the presented TL network is clearly demonstrated. 




FIGURE IV. VOLTAGE DISTRIBUTIONS OF THE EQUIVALENT TL CIRCUIT (A) WITHOUT AND (B) WITH CONCENTRATOR WHEN A 50MHZ POINT SOURCE IS LOCATED AT TOPSIDE. (C) ELECTRIC FIELD DISTRIBUTIONS IN THE VICINITY OF IDEAL CONCENTRATOR SIMULATED BY COMSOL.

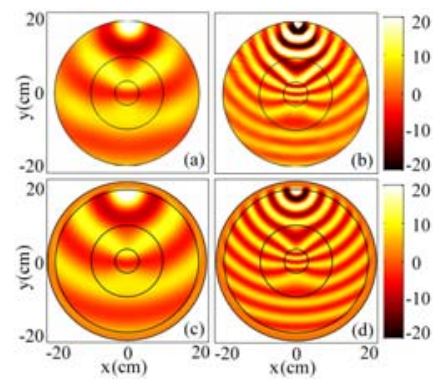

FIGURE V. VOLTAGE DISTRIBUTIONS OF THE EQUIVALENT TL CIRCUIT WORKING AT DIFFERENT FREQUENCIES, (A) F=25MHZ AND (B) F=75MHZ. (C) AND (D) ARE THE CORRESPONDING ELECTRIC FIELD DISTRIBUTIONS OF (A) AND (B) SIMULATED BY COMSOL.

Due to the no resonant nature of TL network usually exhibiting weak dispersion, the broadband characteristic is expected for the proposed concentrator. To confirm this viewpoint, Figs. 5(a) and 5(b) show the voltage distributions of the equivalent TL circuit at frequencies of $25 \mathrm{MHz}$ and $75 \mathrm{MHz}$. The corresponding electric field distributions simulated by COMSOL are also plotted for comparison, as displayed in Figs. 5(c) and 5(d). It is observed that the simulation results for TL and TO-based concentrators agree well with each other. This implies that the cylindrical concentrator realized by TL network can effectively work in a wide frequency range. As a matter of fact, the good performance of TL concentrator can be well extended to a much lower frequency, but we should properly handle the case of a high frequency because the incident wavelength becomes too small compared to the size of the TL unit cell.

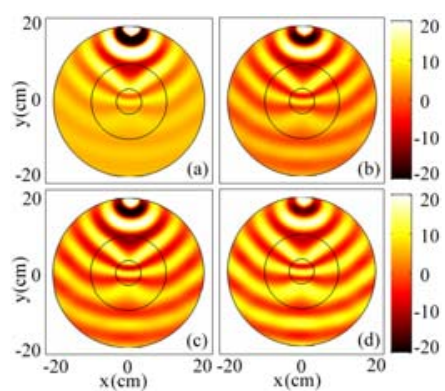

FIGURE VI. VOLTAGE DISTRIBUTIONS OF THE EQUIVALENT TL CIRCUIT WITH DIFFERENT QUALITY FACTORS, (A) $\mathrm{Q}=10$, (B) $\mathrm{Q}=30$, (C) $\mathrm{Q}=50$ AND (D) $\mathrm{Q}=10^{5}$.

Since the material loss of TL network is inevitable in practical applications, it does make sense to study the effect of quality factor of circuit elements on the performance of suggested concentrator. In addition to the afore-discussed case of $\mathrm{Q}=50$ [see Fig. 4(b) or Fig. 6(c)], voltage distributions of the equivalent TL circuit with quality factor of $\mathrm{Q}=10, \mathrm{Q}=30$ and $\mathrm{Q}=105$ are also calculated and shown in panels of (a), (b) and (d) of Fig. 6. Just as expected, the higher value of quality factor we set, the better performance of TL concentrator we get. Moreover, it is clear that the concentrator performs well even in the case of low quality factors. From the above results, we can conclude that the designed TL concentrator is robust against the material loss of circuit elements.

\section{CONCLUSIONS}

In summary, we have proposed an equivalent circuit method to implement cylindrical electromagnetic concentrator based on the TL network. The corresponding TL parameters of the concentrator are derived, and then the method's validity and the device's concentrating effect are confirmed by the ADS simulations. Results show that the developed TL concentrator behaves nearly as perfect as the ideal one and is robust against the material loss of circuit elements. More interestingly, we find that the weak material dispersion resulted from the inherently no resonant property of TL network enables the TL concentrator to operate in a wide bandwidth. It is believed that the method presented here would pave an alternative way for realizing TO-based electromagnetic devices with complicated material parameters.

\section{ACKNOWLEDGEMENTS}

This work was supported by the National Natural Science Foundation of China (Grant Nos. 61161007, 61261002, 61461052), the Fostering Foundation for the Excellent Ph. D. Dissertation of Yunnan University (Grant No. XT512004), and the Sixth Research \& Innovation Project of Graduate Student of Yunnan University (Grant No. ynuy201384).

\section{REFERENCES}

[1] Jiang, W. X., Chin, J. Y. \& Cui, T. J., Anisotropic metamaterial devices. Mater. Today, 12, pp. 26-33, 2009.

[2] Chen, H. Y., Chan, C. T. \& Sheng, P., Transformation optics and metamaterials. Nat. Mater., 9, pp. 387-396, 2010.

[3] Kundtz, N. B., Smith, D. R. \& Pendry, J. B., Electromagnetic design with transformation optics. Proc. IEEE, 99(10), pp. 1622-1633, 2011.

[4] Kildishev, A. V. \& Shalaev, V. M., Transformation optics and metamaterials. Phys. Usp., 54(1), pp. 53-63, 2011.

[5] Liu, Y. M. \& Zhang, X., Recent advances in transformation optics. Nanoscale, 4(17), pp. 5277-5292, 2012.

[6] Rahm, M., Schurig, D., Roberts, D. A., Cummer, S. A., Smith, D. R. \& Pendry, J. B., Design of electromagnetic cloaks and concentrators using form-invariant coordinate transformations of Maxwell's equations. Phot. Nano. Fund. Appl., 6(1), pp. 87-95, 2008.

[7] Lin, L., Wang, W., Du, C. L. \& Luo, X. G., A cone-shaped concentrator with varying performances of concentrating. Opt. Express, 16(10), pp. 6809-6814, 2008.

[8] Yang, J. J., Huang, M., Yang, C. F., Xiao, Z. \& Peng, J. H., Metamaterial electromagnetic concentrators with arbitrary geometries. Opt. Experss, 17(22), pp. 19656-19661, 2009. 
[9] Yu, G. X., Jiang, W. X., Zhou, X.Y. \& Cui, T. J., Non-rotationally invariant invisibility cloaks and concentrators of EM Waves. Eur. Phys. J. Appl. Phys., 44(2), pp. 181-185, 2008.

[10] Li, T. H., Huang, M., Yang, J. J., Mu, S. J. \& Mao, F. C., Three dimensional electromagnetic concentrators with homogeneous material parameters. Progress in Electromagnetics Research M, 18, pp. 119-130, 2011.

[11] Zhang, K., Wu, Q., Fu, J. H. \& Li, L. W., Cylindrical electromagnetic concentrator with only axial constitutive parameter spatially variant. J. Opt. Soc. Am. B, 28(6), pp. 1573-1577, 2011.

[12] Bian, B. R., Liu, S. B., Wang, S. Y., Kong, X. K., Guo, Y. N., Zhao, X., Ma, B. \& Chen, C., Cylindrical optimized nonmagnetic concentrator with minimized scattering. Opt. Express, 21(S2), pp. A231-A240, 2013.

[13] Li, C., Liu, X. \& Li, F., Experimental observation of invisibility to a broadband electromagnetic pulse by a cloak using transformation media based on inductor-capacitor networks. Phys. Rev. B, 81, p. 115133, 2010.

[14] Zedler, M. \& Eleftheriades, G. V., Anisotropic transmission line metamaterials for 2D transformation optics applications. Proc. IEEE, 99(10), pp. 1634-1645, 2011.

[15] Grbic A. \& Eleftheriades, G. V., Periodic analysis of a 2-D negative refractive index transmission line structure. IEEE Trans. Antennas Propag., 51(10), pp. 2604-2611, 2003. 